\title{
INVARIANT CONVOLUTION ALGEBRAS
}

\author{
by J. W. BAKER \\ (Received 21st November 1972)
}

Let $A$ be a commutative, semi-simple, convolution measure algebra in the sense of Taylor (6), and let $S$ denote its structure semigroup. In (2) we initiated a study of some of the relationships between the topological structure of $A^{\wedge}$ (the spectrum of $A$ ), the algebraic properties of $S$, and the way that $A$ lies in $M(S)$. In particular, we asked when it is true that $A$ is invariant in $M(S)$ or an ideal of $M(S)$ and also whether it is possible to characterise those measures on $S$ which are elements of $A$. It appeared from (2) that if $A$ is invariant in $M(S)$ then $S$ must be a union of groups and that $A^{\wedge}$ must be a space which is in some sense "very disconnected". In (3) we showed that if $A^{\wedge}$ is discrete then $A$ is "approximately" an ideal of $M(S)$. (What is meant by "approximately" is explained in (3); it is the best one can expect since algebras which are approximately equal have identical structure semigroups and spectra.) In this paper we round off some of the results of (2) and (3). We show that if $A$ is invariant in $M(S)$ then $A^{\wedge}$ is totally disconnected, and that if $A^{\wedge}$ is totally disconnected then $S$ is an inverse semigroup (union of groups). From these two crucial facts it is fairly straight-forward to obtain a complete characterisation of algebras $A$ (and their structure semigroups) for which (i) $A^{\wedge}$ is totally disconnected, (ii) $A$ is invariant in $M(S)$, or (iii) $A$ is an ideal of $M(S)$. This is done for (i) and (ii) in the latter half of this paper (Theorem 1, and after). The former of the two facts mentioned above enables us to concentrate our attention upon the idempotents of $A$, so that we can make use of Taylor's generalisation of Cohen's idempotent Theorem (see (8), Theorem B). The fact that $S$ is a union of groups allows us to make use of the well-behaved duality theory of compact and discrete inverse semigroups. Throughout this paper we shall use the notation and terminology of (3) without explanation.

We begin with a number of fairly simple lemmas.

Lemma 1. Let $S$ be a compact, commutative inverse semigroup for which $S^{\wedge}$ separates the points of $S$. Let $T$ be a proper closed subsemigroup of $S$. Either there exist distinct continuous semicharacters $\chi_{1}$, and $\chi_{2}$ such that $\chi_{1}$ agrees with $\chi_{2}$ on $T$ or there exists $\chi_{3} \in S^{\wedge}$ which vanishes on $T$.

Proof. Let $S_{1}$ denote the semigroup consisting of $S$ with an isolated identity adjoined, and let $T_{1}$ denote $T$ together with the identity of $S_{1}$. Clearly $S_{1}$ separates the points of $S_{1}$, and $S_{1}$ and $T_{1}$ are each compact inverse semigroups with identity. Now every continuous semicharacter of $T_{1}$ can be extended to an element of $S_{1}^{\wedge}$ (see (5), Corollary 2). Suppose that every such extension 
were unique. Then we could identify the discrete inverse semigroups $S_{\hat{1}}$ and $T_{1} \hat{1}$. The Pontrjagin duality theorem for compact inverse semigroups (see (1), Theorem 6.1) tells us that every semicharacter of $T_{\hat{1}} \hat{\text { is generated by an element }}$ of $T_{1}$. But every element of $S_{1}$ generates an element of $T_{1}^{\wedge}{ }^{\wedge}=S_{1}^{\wedge}$. This would imply that $S_{1}=T_{1}$. So we can find $\chi_{1}$ and $\chi_{2}$ in $S_{1}^{\hat{1}}$ with $\chi_{1} \neq \chi_{2}$ but $\chi_{1}=\chi_{2}$ on $T_{1}$. If we think of $\chi_{1}$ and $\chi_{2}$ as functions on $S$ then $\chi_{1}$ is still distinct from $\chi_{2}$. If $\chi_{1} \equiv 0$ on $S$ then $\chi_{2} \neq 0$ on $S$, so $\chi_{2} \in S^{\wedge}$. Also $\chi_{2}=\chi_{1}$ on $T \subseteq S$ so $\chi_{2} \equiv 0$ on $T$. So we can take $\chi_{3}=\chi_{2}$. Likewise if $\chi_{2} \equiv 0$ on $S$ ! If neither of these cases is true then $\chi_{1}$ and $\chi_{2}$ are distinct elements of $S^{\wedge}$ agreeing on $T$, as required.

Lemma 2. Let $A$ be a CMA with structure semigroup $S$. If $A$ is invariant in $M(S)$ (that is, $\mu * \bar{x} \in A$ whenever $\mu \in A$ and $x \in S$ ) then $A^{\wedge}$ (the spectrum of $A$ ) is totally disconnected.

Proof. By Lemma 3.2 of (2) we know that $S$ is an inverse semigroup. Let $e \in E_{g}$ (the set of generating idempotents of $S$ ). Then $S(e)$ is an open and closed subsemigroup of $S$. We can find a probability measure $\mu$ in $A$ with support contained in $S(e)$. Then $\mu * \bar{e}$ is a probability measure of $A$ with support contained in $G(e)$. Let $A_{e}$ denote the set of measures in $A$ which have their support contained in $G(e)$. (We shall sometimes think of $A_{e}$ as a subset of $M(G(e)$ ).) If $e$ is in $E_{g}$ then $A_{e} \neq\{0\}$. By Theorem 3 of (7), there exists a locally compact group $H(e)$, which is (algebraically) a subgroup of $G(e)$, which has Bohr compactification $G(e)$, for which $L^{1}(H(e)) \subseteq A_{e} \subseteq\left(L^{1}(H(e))\right)^{\frac{1}{2}}$. (We think of $A_{e}$ as a subalgebra of $M(H(e))$. Then $\left(L^{1}(H(e))\right)^{\frac{1}{2}}$ denotes the Jacobson radical in $M(H(e))$ of $L^{1}(H(e))$. Alternatively, it is the closure of $L^{1}(H(e))$ in $M(H(e))$ for the spectral radius norm (cf. (2) and (3)). Since $A_{e}$ is invariant in $M(G(e)$ ) it is clear that $H(e)=G(e)$. Therefore $H(e)$ is equal to $G(e)$ topologically (cf. (4), Corollary 2.4). So $A \supseteq L^{\mathbf{1}}(G(e))$ for each generating idempotent $e$ of $S$.

Let $\chi$ and $\chi^{\prime}$ be distinct elements of $S^{\wedge}$, and let $|\chi|=\chi_{e},\left|\chi^{\prime}\right|=\chi_{f}$, where $e$ and $f$ are generating idempotents of $S$. First consider the case in which $e=f$. If $\mu$ denotes normalised Haar measure on $G(e)$ then $v=\bar{\chi} \cdot \mu$ (i.e. $\left.\int f(x) d v(x)=\int f(x) \overline{\chi(x)} d \mu(x)\right)$ is an idempotent of $A$. Also, $\int \chi(x) d v(x)=1$ and $\int \chi^{\prime}(x) d v(x)=0$, since $\chi \not \equiv \chi^{\prime}$ on $G(e)$ and so $\chi^{\prime} \chi \not \equiv 1$ on $G(e)$. So $\chi$ and $\chi^{\prime}$ are in different components of $A^{\wedge}$ (identified with $S^{\wedge}$ ). Suppose now that $e \neq f$, say $e \geq f$. Then $\chi^{\prime}=0$ on $G(e)$. Let $\mu$ and $\nu$ be as above, then $\int \chi(x) d v(x)=1$ and $\int \chi^{\prime}(x) d v(x)=0$, as before. So $A^{\wedge}$ is totally disconnected.

Lemma 3. Let $A$ be a CMA with structure semigroup $S$. If $A^{\wedge}$ is totally disconnected then $S$ is an inverse semigroup.

Proof. This is a modification of the proof of Theorem 2.6 of (2). If $A^{\wedge}$ is totally disconnected then under the norm topology (as a subset of $A^{\prime}$ ) $A^{\wedge}$ contains no non-trivial connected subsets. If $S$ is not an inverse semigroup there will exist $\chi$ in $S^{\wedge}$ and $x$ in $S$ so that $0<|\chi(x)|<1$. For each $\alpha \geqq 1$ let $\chi_{\alpha}$ 
denote the semicharacter $|\chi|^{z}$. Then the map $\alpha \rightarrow \chi_{\alpha}$ is a continuous injection of $[1, \infty)$ into $S^{\wedge}$ where $S^{\wedge}$ has the topology of uniform convergence on $S$. Since this topology is equal to the norm topology of $A^{\wedge}=S^{\wedge}$, we have established the required contradiction.

Lemma 4. Let $S$ be a compact commutative inverse semigroup for which $S^{\wedge}$ separates the points of $S$. Let $A$ be a convolution measure algebra contained in $M(S)$. Suppose that the natural map $\chi \rightarrow h$, where $h(\mu)=\int \chi(x) d \mu(x)$ for $\mu$ in $A$, of $S^{\wedge}$ into $A^{\wedge}$ is an injection. Then the carrier of $A$ is the whole of $S$, that is $A$ is $\sigma(M(S), C(S))$-dense in $M(S)$. (This means that if $A^{\wedge}$ is naturally identified with $S^{\wedge}$ then $S$ is the structure semigroup of $A$.)

Proof. Let $T$ denote the carrier of $A$. So $T$ is the closure of the union of the supports of measures in $A$. Clearly $T$ is a closed subsemigroup of $S$. Suppose that $T \neq S$. We can apply Lemma 1 to $S$ and $T$. Let $\chi_{3}$ vanish on $T$. Then $h(\mu)=\int \chi_{3}(x) d \mu(x)=0$ for all $\mu$ in $A$. So $h \notin A^{\wedge}$. Let $\chi_{1}=\chi_{2}$ on $T$. Then $\int \chi_{1}(x) d \mu(x)=\int \chi_{2}(x) d \mu(x)$ for all $\mu$ in $A$. Either of these possibilities contradicts the fact that the natural map is a bijection of $S^{\wedge}$ into $A^{\wedge}$. So we have the required contradiction.

Lemma 5. If $A$ is a CMA with structure semigroup $S$ and if $A$ is invariant in $M(S)$ then $A^{\frac{1}{2}}$ is also invariant in $M(S)$.

Proof. Since $A^{\frac{1}{t}}$ denotes the closure of $A$ in $M(S)$ for the spectral radius norm, and since that norm is an algebra norm on $M(S)$, this lemma is obvious.

Let $S$ be a topological inverse semigroup in which $S$ separates the points of $S$. We can construct what we shall call the Bohr compactification of $S$. Let $S^{\prime}$ denote the set of all continuous homomorphisms of $S$ into the disc semigroup $\left(S^{\prime}=S^{\wedge} \cup\{0\}\right)$. Then $S^{\prime}$ can be thought of as a discrete semigroup, under pointwise multiplication. Let $b(S)$ denote the closure of the semigroup $S$ in the compact inverse semigroup $\left(S^{\prime}\right)^{\wedge}$. Then, by virtue of the Pontrjagin duality theorem for discrete inverse semigroups ((1), Theorem 5.16), one can easily see that $b(S)$ is a compact inverse semigroup which has plenty of continuous semicharacters, which contains $S$ as a dense subsemigroup, and for which every continuous semicharacter on $S$ (in its original topology) extends to an element of $b(S)^{\wedge}$. In particular, we can identify $S^{\wedge}$ and $b(S)^{\wedge}$. It is clear from the Pontrjagin duality theorem for compact inverse semigroups that $b(S)$ is uniquely characterised by the properties listed above.

Lemma 6. Let $S$ be a locally compact inverse semigroup in which $S^{\wedge}$ separates the points of $S$, and let $b(S)$ denote its Bohr compactification. If e is a generating idempotent of $S$ then e is a generating idempotent of $b(S)$. Conversely, if $S$ is an ideal of $b(S)$ then every generating idempotent of $b(S)$ is a generating idempotent of $S$.

Proof. Let $e$ be a generating idempotent of $S$; that means that $\chi_{e}$ is in $S^{\wedge}$. Let $\chi$ be the continuous semicharacter of $b(S)$ which extends $\chi_{e}$. Then there 
exists $f$, a generating idempotent of $b(S)$, such that $\chi=\chi_{s}$. We must show that $e=f$. Since $\chi_{f}(e)=\chi(e)=\chi_{e}(e)=1$, it is clear that $e \geqq f$. Since $S$ is dense in $b(S)$ we can find a set $\left\{e_{\alpha}\right\}$ of idempotents of $S$ converging to $f$. Then $\chi_{e}\left(e_{\alpha}\right)=\chi_{f}\left(e_{\alpha}\right) \rightarrow \chi_{f}(f)=1$, so $\chi_{e}\left(e_{\alpha}\right)=1$ eventually. Therefore $e_{\alpha} \geqq e$ eventually, so that $f \geqq e$. This shows that $e$ is generating in $S$.

Suppose that $S$ is an ideal of $b(S)$, and that $f$ is a generating idempotent of $b(S)$. Choose a set of idempotents $\left\{e_{\alpha}\right\}$ in $S$ converging to $f$. Since $f$ is generating, $e_{\alpha} \geqq f$ eventually. So $f=f e_{\alpha}$ is in $S$. Since the element $\chi_{f}$ of $b(S)^{\wedge}$ is the extension of an element of $S^{\wedge}$ it is clear that $f$ is generating in $S$.

We remark that if $S$ is the set of natural numbers in the discrete topology with minimum as its binary operation then $S$ becomes a non-trivial ideal of $b(S)$.

The preliminary lemmas are now complete, and we turn to our characterisations of CMA's and their structure semigroups. If $X$ is a locally compact topological space then we shall say that a complex function on $S$ is simple if it is continuous, has finite range, and has compact support. If $X$ is totally disconnected then the simple functions on $X$ are uniformly sense in $C_{0}(X)$. If $A$ is a CMA with structure semigroup $S$ we shall denote by $R_{A}(S)$ the closure in $M(S)$ of the set of measures on $S$ whose Gelfand transform is a simple function on $S^{\wedge}$. (We are identifying $S^{\wedge}$ with a subset $M(S)^{\wedge}$ in the natural way and giving it the Gelfand topology $\sigma\left(S^{\wedge}, A\right)=\sigma\left(A^{\wedge}, A\right)$.) We shall characterise this space later. We shall use $A_{1}$ to denote the closure in $M(S)$ of the linear span of the algebras $L^{1}(G)$ where $G$ is a compact subgroup of $S$ and the Haar measure on $G$ is in $A$. We also remind the reader that if $B$ is a subalgebra of $M(S)$ then $B_{s}^{+}$(or just $B^{\frac{1}{2}}$ ) denotes the closure of $B$ in $M(S)$ for the spectral radius norm

$$
v(x)=\sup \{|h(x)|: h \text { is a complex homomorphism on } M(S)\} .
$$

Lemma 7. Let $A$ be a CMA with totally disconnected spectrum and structure semigroup $S$. Then $R_{A}(S)=A_{1} \subseteq A$. Further, $R_{A}(S)$ is a CMA.

Proof. That $R_{A}(S) \subseteq A$ is immediate from Silov's idempotent theorem. It is clear that $R_{A}(S)$ is the closed linear span of the idempotent measures of $A$, by the same theorem. By Theorem B of (8) it is clear that every idempotent measure of $A$ is in the linear span of the measures in $A$ which are absolutely continuous with respect to idempotent probability measures in $A$. Since every idempotent probability measure on $S$ is the Haar measure of some compact subgroup of $S$, it is clear that $R_{A}(S) \subseteq A_{1}$.

If $\mu$ is in $R_{A}(S)$ and $\chi \in S^{\wedge}$ the measure $\chi . \mu$ is in $A$. So $(\chi \cdot \mu)^{\sim}$ is in $C_{0}\left(A^{\wedge}\right)$. If $\mu^{\sim}$ were simple then $(\chi . \mu)^{\sim}$ would also be simple and so would be an element of $R_{A}(S)^{\sim}$. Since $R_{A}(S)$ is closed it follows that $\chi \cdot \mu$ is in $R_{A}(S)$ for all $\mu$ in $R_{A}(S)$ and all $\chi \in S^{\wedge}$, and again, since $R_{A}(S)$ is closed, $R_{A}(S)$ is a convolution measure algebra. From this fact it follows that every measure in $A_{1}$ is an element of $R_{A}(S)$. Therefore $A_{1}=R_{A}(S)$.

We now obtain an analogue of Theorem 1 of (3). 
Theorem 1. Let $A$ be a CMA with structure semigroup $S$ and totally disconnected spectrum. Then $R_{A}(S) \subseteq A \subseteq\left(R_{A}(S)\right)^{\frac{1}{2}}$. Further, both $R_{A}(S)$ and $\left(R_{A}(S)\right)^{\frac{1}{t}}$ are CMA's with structure semigroup $S$ and totally disconnected spectrum.

Proof. Let $\hat{\mu} \in A$. By Silov's idempotent theorem we may approximate $\mu$ in the spectral radius norm by a sequence of measures in $A$ which have simple Gelfand transforms on $A^{\wedge}$. Therefore $R_{A}(S) \subseteq A \subseteq\left(R_{A}(S)\right)^{\frac{1}{2}}$. Since $R_{A}(S)$ is dense in $A$ for the spectral radius norm, $R_{A}(S)^{\wedge}=A^{\wedge}=S^{\wedge}$. So, by Lemma 4, it is clear that $S$ is the structure semigroup of $R_{A}(S)$.

If $\mu$ is in $\left(R_{A}(S)\right)^{\frac{1}{2}}$ we can choose a sequence $\left\{\mu_{n}\right\}$ in $A$ converging to $\mu$ in the spectral radius norm. If $\chi \in S$ then $\left\{\chi \cdot \mu_{n}\right\}$ converges to $\chi \cdot \mu$ in this norm. Since $\chi \cdot \mu_{n}$ is in $A$ is is clear that $\chi \cdot \mu \in\left(R_{A}(S)\right)^{\frac{1}{t}}$. Since $\left(R_{A}(S)\right)^{\frac{1}{1}}$ is closed in $M(S)$ it is a convolution measure algebra; its structure semigroup is obviously equal to $S$. Finally, $\left(R_{A}(S)\right)^{\wedge}$ is totally disconnected because $R_{A}(S)$ is the closed linear span of its idempotents. It follows that $\left(R_{A}(S)^{\frac{1}{2}}\right.$ has totally disconnected spectrum.

Corollary 1. If $A$ and $S$ are as in Theorem 1 then $A^{\frac{1}{2}}=\left(R_{A}(S)\right)^{\frac{1}{2}}$.

Corollary 2. Let $A$ and $B$ be CMA's with structure semigroup $S$. If their spectra induce the same totally disconnected topology onto $S^{\wedge}$ then

$$
R_{A}(S) \subseteq B \subseteq\left(R_{A}(S)\right)^{\frac{1}{2}}
$$

The above corollaries follow immediately from the theorem. We state a third corollary which is best established after Theorem 2.

Corollary 3. Let $A$ be a CMA with structure semigroup $S$ and totally disconnected spectrum. There exists a locally compact inverse semigroup $S_{1}$ such that $S$ is the Bohr compactification of $S_{1}$ and $R_{A}(S)$ is the closure in $M(S)$ of the algebra

$$
\Sigma\left\{L^{1}\left(G_{1}(e)\right): e=e^{2} \in S_{1}\right\},
$$

where $G_{1}(e)$ denotes the maximal subgroup of $S_{1}$ containing $e$, and $G_{1}(e)$ is open and closed in $S_{1}$.

Theorem 2. Let $S$ be a compact, Hausdorff, commutative inverse semigroup. In order that $S$ be the structure semigroup of a CMA with totally disconnected spectrum it is necessary and sufficient that $S$ be the Bohr compactification of a locally compact, commutative, inverse semigroup $S_{1}$ in which every maximal subgroup is open and closed and has totally disconnected dual group.

Proof. Let $A$ be a CMA with $A^{\wedge}$ totally disconnected, and let $S$ denote its structure semigroup. By virtue of Theorem 1, we may assume that $A=R_{A}(S)$. For each idempotent $e$ of $S$ let $A_{e}$ denote the set of measures in $A$ whose support is contained in $G(e)$. Arguing as in Lemma 2 we see that if $A_{e} \neq\{0\}$ then $L^{1}(H(e)) \subseteq A_{e} \subseteq\left(L^{1}(H(e))\right)^{\frac{1}{2}}$ where $H(e)$ is a group whose Bohr compactification is $G(e)$. Since $A_{1}=R_{A}(S)$ and $\left(L^{1}(H(e))\right)^{\frac{1}{2}} / L^{1}(H(e))$ contains no idempotents, it is clear that $A_{e}=L^{1}(H(e))$. Since every idempotent probability 
measure has for its support a compact subgroup of $S$, it is clear that $A=A_{1}$ is the closed linear span of the algebras $L^{1}(H(e))$. So $A$ is the closure of $\Sigma\left\{L^{1}(H(e)): e \in E_{1}\right\}$ where $E_{1}$ is the set of idempotents of $S$ for which $A_{e} \neq\{0\}$. Clearly $E_{1}$ is a subsemigroup of $E$. We must have that

$$
L^{1}(H(e)) L^{1}(H(f)) \subseteq L^{1}(H(e f))
$$

for each $e, f \in E_{1}$, since $A$ is an algebra. It follows that $H(e) . H(f) \subseteq H(e f)$, so that the set $S_{1}=\left\{H(e): e \in E_{1}\right\}$ is a subsemigroup of $S$. Since $A_{1}$ is the convolution measure algebra generated by the idempotent probability measures of $A$ (cf. proof of Lemma 7) it is clear that, for each $e$ in $E_{1}, A_{e}$ is the convolution measure algebra generated by its idempotent probability measures. Therefore $H(e)^{\wedge}$ is totally disconnected. Each $H(e)$ has already been topologised as a locally compact group; topologise $S_{1}$ so that each $H(e)$ is open and closed in $S_{1}$. The multiplication on $S$ is continuous from $G(e) \times G(f)$ to $G(e f)$, for each $e$ and $f$ in $E$. Let $e$ and $f$ be in $E_{1}$. The multiplication is continuous from

$$
b(H(e) \times H(f))=b(H(e)) \times b(H(f))
$$

to $G(H(e f))$, so is weakly continuous from $H(e) \times H(f)$ to $H(e f)$. By Corollary 2.1 of (4), we conclude that the multiplication of $S_{1}$ is continuous from $H(e) \times H(f)$ to $H(e f)$. So $S_{1}$ is a locally compact, topological inverse semigroup in which $S_{1} \hat{~ s e p a r a t e s ~ t h e ~ p o i n t s ~ o f ~} S_{1}$. So we can consider the Bohr compactification $T$ of $S_{1}$. The necessity of the condition in the theorem will be established if we show that $T$ is the structure semigroup of $A$. Since $A$ is just the completion of a subalgebra of $M\left(S_{1}\right)$, we can regard $A$ as a subalgebra of $M(T)$. It is clear that $A$ becomes an $L$-subalgebra of $M(T)$ with carrier space $T$. Every continuous semicharacter of $S_{1}$ will define a non-zero complex homomorphism of $A$; distinct semicharacters of $S_{1}$ will define distinct complex homomorphisms of $A$. Let $h \in A^{\wedge}$. For each idempotent $e \in S_{1}$ we can find $\chi$, either zero or an element of $H(e)^{\wedge}$ such that $h(\mu)=\int \chi(x) d \mu(x)$ for all $\mu$ in $A_{e}=L^{1}(H(e))$. In this way we define a continuous semicharacter $\chi$ of $T$. Then $h(\mu)=\int \chi d \mu$ for all $\mu$ in $A$. So $T$ is the structure semigroup of $A$. This establishes the necessity of the condition.

Let $S_{1}$ be a semigroup as in the statement of the theorem. We let $A$ be the closure in $M(S)$ of the algebra $\Sigma\left\{L^{\prime}(H(e)): e=e^{2} \in S_{1}\right\}$, where $H(e)$ is the maximal subgroup of $S_{1}$ containing $e$. Then $A$ is a CMA. Arguing as above, we see that $A^{\wedge}=S^{\wedge}=S_{1}^{\wedge}$, so that $S$ is the structure semigroup of $A$. Since each maximal subgroup $H(e)$ of $S_{1}$ has totally disconnected dual it is clear that the idempotents of $L^{1}(H(e))$ are dense in $L^{1}(H(e))$ for the spectral radius norm. Therefore, the idempotents of $A$ are dense for this norm so that $A^{\wedge}$ is totally disconnected. This completes the proof of the theorem.

Proof of Corollary 3. This can be proved using the first half of the proof of Theorem 2. We showed there that if $R_{A}(S)=A$ then the conclusions of this corollary are valid.

We now turn to a characterisation of invariant CMA's. 
Theorem 3. Let $S$ be a compact Hausdorff, commutative topological semigroup. Then $S$ is the structure semigroup of a CMA which is invariant in $M(S)$ if and only if

(i) $S$ is the Bohr compactification of $S_{1}=\left\{G(e): e \in E_{g}\right\}$ where each subgroup $G(e)$ of $S_{1}$ is open and closed and has the relative topology as a subset of $S$;

(ii) $E_{g}$ is an ideal of $E$; and

(iii) if $e \in E_{g}$ and $f$ is an idempotent with $f<e$ then the quotient group $G(f) / f G(e)$ is finite.

Proof. This is similar to the proof of Theorem 2. Let $A$ be a CMA with structure semigroup $S$ and suppose $A$ is invariant in $M(S)$. We know that $A^{\wedge}$ is totally disconnected and that $S$ is a union of groups. If $A_{e} \neq\{0\}$ then $L^{1}(H(e)) \subseteq A_{e} \subseteq\left(L^{1}(H(e))\right)^{\frac{1}{2}}$ where $H(e)$ has Bohr compactification $G(e)$. Now $A_{e}$ is invariant in $M(G(e))$. It follows that $H(e)=G(e)$, so $H(e)$ is the compact group $G(e)$ (cf. Corollary 2.4 of (4)). Therefore, as in Theorem 2, we can assume that $A$ contains the closure of $\Sigma\left\{L^{1}(G(e)): e \in E_{1}\right\}$, where $E_{1}$ is a subsemigroup of $E, E_{1}=\left\{e \in E: A_{e} \neq\{0\}\right\}$. Since $A$ is invariant, $E_{1}$ is an ideal of $E$. As in Theorem 2 we have that $S$ is the Bohr compactification of

$$
S_{1}=\bigcup\left\{G(e): e \in E_{1}\right\}
$$

where $G(e)$ is open and closed in $S_{1}$. It follows from Lemma 6 that $E_{1}$, which is the set of generating idempotents of $S_{1}$, is equal to the set $E_{\theta}$ of generating idempotents of $S$. So (i) and (ii) are established.

Now if $e \in E_{g}$ and $f<e$ is an idempotent then $\mu_{e}$ (the Haar measure on $G(e)$ ) is in $A$. So $\mu_{e}^{* f}$ is in $A_{f}$. But $\mu_{e} * \bar{f}$ is the Haar measure on $f G(e)$, so the Haar measure on $f G(e)$ is in $\left(L^{1}(G(f))\right)^{\frac{1}{2}}$. It follows that $f G(e)$ has positive Haar measure in $G(f)$, that is that $G(f) / f G(e)$ is finite.

Conversely, let $S$ and $S_{1}$ satisfy (i), (ii) and (iii). Let $A$ be the closure in $M(S)$ of $\Sigma\left\{L^{1}(G(e)): e \in E_{g}\right\}$. From (ii) and (iii) it is clear that $A$ is invariant in $M(S)$. The proof of Theorem 2 shows that $A$ is a CMA with structure semigroup $S$.

Corollary. Let $A$ be a CMA with structure semigroup $S$. Suppose that $A$ is invariant in $M(S)$. Then $E_{g}$ is an ideal of $E$. Also $R_{A}(S)=R(S)=L^{1}(S)$ (the closure in $M(S)$ of $\Sigma\left\{L^{1}(G(e))\right.$ : $\left.\left.e \in E_{g}\right\}\right)$. Further $R(S)$ and $(R(S))^{\frac{1}{2}}$ are invariant subalgebras of $M(S)$.

Proof. All except the last sentence is clear from Theorem 3 and Corollary 3 to Theorem 1. That $R(S)=L^{1}(S)$ is invariant in $M(S)$ is shown in the proof of Theorem 3. To complete the proof we apply Lemma 5.

In the introduction we discussed the question as to when $A$ is an ideal of $M(S)$. In that case it must be invariant, so that the above conditions are necessary. However, the distinction between the cases in which $A$ is respectively 
invariant or an ideal would seem to depend upon the existence, or non-existence, of certain pathological measures. Difficulties may arise if, for $e$ is $E_{g}$, the space $e E$ can contain continuous measures. If this is so, then one needs some extra condition which prevents this from happening in order to characterise algebras, and their structure semigroups, which are ideals in $M(S)$. We omit the details because of the pathological behaviour of the problem.

\section{REFERENCES}

(1) C. W. Austin, Duality theory for commutative semigroups, Trans. Amer. Math. Soc. 109 (1963), 245-256.

(2) J. W. BAKER, Convolution algebras with discrete spectra, J. London Math. Soc. (2) 5 (1972), 193-201.

(3) J. W. BAKER, A characterisation of a certain class of convolution algebras, Proc. Edinburgh Math. Soc. (2) 18 (1973), 199-205.

(4) I. GLICKsBerg, Uniform boundedness for groups, Canad. J. Math. 14 (1962), 269-276.

(5) T. P. Srinivasan and U. B. Tewari, A Stone-Weierstrass theorem for semigroups, Bull. Amer. Math. Soc. 74 (1968), 1125-1127.

(6) J. L. TAYLor, The structure of convolution measure algebras, Trans. Amer. Math. Soc. 119 (1965), 150-166.

(7) J. L. TAYLOR, L-subalgebras of $M(G)$, Trans. Amer. Math. Soc. 135 (1969), 105-113.

(8) J. L. TAYLOR, The cohomology of the spectrum of a measure algebra, Acta Math. 126 (1971), 195-225.

UNIVERSITY OF SHEFFIELD 